\section{Postoperative change in lateral rectus muscle insertion measured by anterior segment optical coherence tomography}

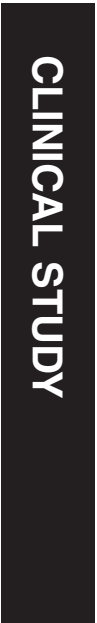

${ }^{1}$ Department of

Ophthalmology, Samsung

Medical Center,

Sungkyunkwan University

School of Medicine, Seoul, Korea

${ }^{2}$ Department of Ophthalmology, Eulji Medical Center, Eulji University School of Medicine, Seoul, Korea

Correspondence: SY Oh, Department of Ophthalmology, Samsung Medical Center, Sungkyunkwan University, School of Medicine, \#81 Irwon-ro, Gangnam-gu, Seoul 06351, Korea Tel: +82 23410 3566; Fax: +82 234100029 . E-mail: syoh@skku.edu

Received: 11 September 2016

Accepted in revised form: 29 March 2017

Published online:

16 June 2017 for understanding postoperative changes in EOM after tissue swelling subsides. Eye (2017) 31, 1556-1561; doi:10.1038/eye.2017.89; published online 16 June 2017

Intermittent exotropia, which is commonly observed in patients with strabismus, recurs in

\begin{abstract}
Aims The aims of this study were to investigate the longitudinal change in lateral rectus (LR) muscle insertion after recession surgery, and to evaluate a relationship between insertion distance and postoperative amount of deviation.

Methods We recruited 31 patients who underwent primary LR recession surgery with normal anterior segment structures. An AS-OCT scan of the LR muscle was performed at every visit. Data on sex, age, degree of deviation (prism diopter), and spur-LR insertion distance using AS-OCT were collected at preoperatively and postoperative months 1, 3, and 6. Spur-LR insertion was defined as the shortest distance between the insertion of the LR muscle and the scleral spur was measured using the caliper function in the AS-OCT software.

Results The mean distance of spur-LR insertion measured with AS-OCT was $5.5 \pm 0.7 \mathrm{~mm}$ preoperatively, and $11.1 \pm 0.9 \mathrm{~mm}$ at 1 month, $11.5 \pm 0.8 \mathrm{~mm}$ at 3 months, and $11.0 \pm 0.7 \mathrm{~mm}$ at 6 months postoperatively. There were significant differences in spur-LR insertion between postoperative 1 and 3 months, and between 3 and 6 months (generalized estimating equation, all $P<0.001$ ). The overall spur-LR insertion measurement has a significantly negative correlation with postoperative amount of deviation (Pearson's correlation, $P=0.035, r=-0.218$ ).

Conclusions AS-OCT is a useful instrument

$\sim 30 \%$ of patients who undergo strabismus surgery. ${ }^{1,2}$ Several studies have suggested movement of new muscle, as a possible cause of this recurrence. As a result there have been many trials investigating changes in the muscle insertion site after strabismus surgery. ${ }^{1-3}$ Most previous studies evaluated the site of new muscle insertion through animal studies, and reported that the new attachment site of muscle insertion slowly crept forward after recession surgery, especially after surgeries that used the hang-back technique. ${ }^{4}$ However, there have been few studies investigating the postoperative change in muscle insertion in humans, which necessitates the use of non-invasive methods.

The technique of anterior segment optical coherence tomography (AS-OCT) was recently introduced. This approach is non-invasive and allows visualization of the anterior structure of the eye with high resolution. Liu $e t a l^{5}$ first reported that AS-OCT can be used to image the structure of the horizontal rectus muscles, and shows good agreement with intraoperative measurements. Ngo et al ${ }^{6}$ also demonstrated that AS-OCT could accurately detect rectus muscle insertions. However, the change in new muscle insertion after strabismus surgery, as measured with AS-OCT has not been reported. Hence, the purpose of this study were to investigate the longitudinal change in LR muscle insertion after strabismus surgery, and to determine the effect of insertion movement on the angle of deviation.
\end{abstract}

\section{Introduction}

\section{Materials and methods}

This study was a retrospective observational study of patients who were operated on by one experienced surgeon (S.O), who performed primary surgeries at Samsung Medical Center between March and September 2014. This study was approved by the ethics committee of 
Samsung Medical Center Institutional Review Board. The research followed the tenets of the Declaration of Helsinki.

We recruited 31 patients who underwent primary unilateral or bilateral lateral rectus (LR) muscle recession surgery with normal anterior segment structures.

Recession surgery included only conventional methods. In cases of bilateral LR recession, the amount of correction was same in both eyes. The extent of surgery was based on the surgical tables presented in Table 1. We excluded patients who had eyes with anatomical abnormalities such as sclerocornea, corneal pannus, and microcornea, and patients with a history of ocular surgery, significant symblepharon formation, or cicatricial conjunctival changes, all of which may interfere with imaging.

\section{AS-OCT measurements}

All Visante AS-OCT (Carl Zeiss Meditec, Dublin, CA, USA) were performed by one experienced operator (YK). Before surgery, an AS-OCT scan of the lateral rectus muscles was performed. The patients were in a sitting position, and were required to make the maximal gaze guided by a fixation light to scan the face of the muscle insertion. An extension covered the corneal limbus and the insertion of the lateral rectus muscles was scanned by an axial cross-section. The scanning plane $\left(0-180^{\circ}\right)$ was oriented parallel to the long axis of the muscle, and the highest quality image was selected for analysis. In the cross-section image, the end of the cleft between the lateral rectus muscle and sclera was defined as the posterior face of the insertion site.

Preoperatively, we measured the insertion distance of LR muscle using AS-OCT (Figure 1). Spur-LR insertion, described by Park et al, ${ }^{7}$ was the shortest distance between the insertion of LR muscle and scleral spur, and was used for evaluating longitudinal changes in the insertion site after surgery. With the help of the caliper function in the AS-OCT software, we were able to measure spur-LR insertion distance. Image analyses were performed by two independent examiners (IL and JL). Two observers, who were blinded to follow-up date, performed the measurements simultaneously for all data, then measured again in a month after the first measurements. The mean value of the two measurements was used for analysis.

For analysis of longitudinal changes, we used a single value for spur-insertion distance in each eye. For analysis of the relationship between distance measurements and angle of deviation, we selected spur-LR insertion distance of the right eye at random in bilateral LR recession, and used a single value for spur-insertion distance in cases of unilateral LR recession.
Table 1 Surgical dosage for intermittent exotropia patients

\begin{tabular}{llc}
\hline Prism diopters & Laterality & Lateral rectus recession $(\mathrm{mm})$ \\
\hline 15 & Unilateral & 8.5 \\
20 & Unilateral & 9.0 \\
25 & Bilateral & 6.5 \\
30 & Bilateral & 7.0 \\
35 & Bilateral & 7.5 \\
40 & Bilateral & 8.0 \\
50 & Bilateral & 9.0 \\
\hline
\end{tabular}

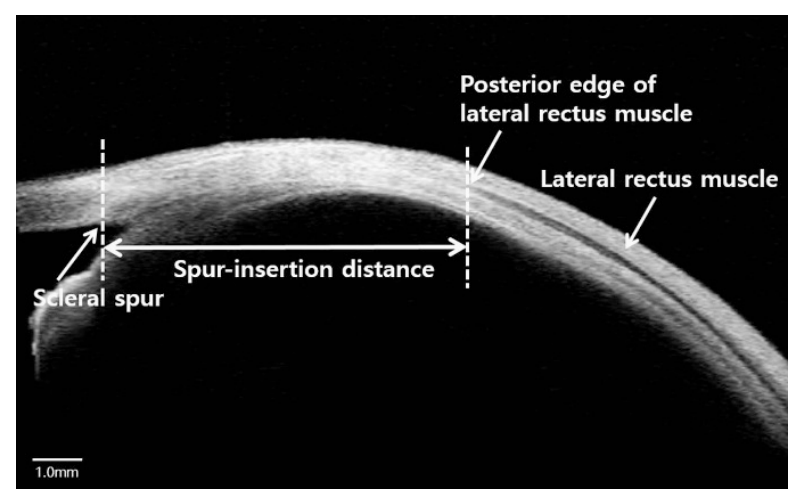

Figure 1 The method for measuring the lateral rectus muscle insertion distance. It is defined as the spur-LR insertion distance, which is the shortest distance between the insertion of lateral rectus muscle and scleral spur.

\section{Postoperative follow-up}

Follow-up examinations were performed at postoperative months 1, 3, and 6. Data on sex, age, degree of deviation (PD) at distance measured by alternate prism cover test, and spur-LR insertion distance measured using AS-OCT were collected (Figure 2). All patients underwent complete ophthalmic examination including prism and alternate cover tests, which were carried out at distances of $6 \mathrm{~m}$ in nine cardinal gaze directions and $30 \mathrm{~cm}$ in the primary gaze. All deviations were determined using the appropriate spectacle correction. We used distance angle of deviation at far for analysis in this study.

Statistical analyses were performed with SPSS version 20 for Windows (SPSS, Inc., Chicago, IL, USA). Paired- $t$ test was used to evaluate the change in muscle insertion site and angle of deviation between each visit. Pearson's correlation were used to evaluate the correlation between the change in spur-LR insertion distance and the change in angle of deviation. We measured intra-observer and inter-observer variability for AS-OCT measurements using intraclass correlation coefficient analysis (ICC). Statistical differences were considered significant when the $P$ value was less than 0.05 . Results are expressed as mean \pm SD. 

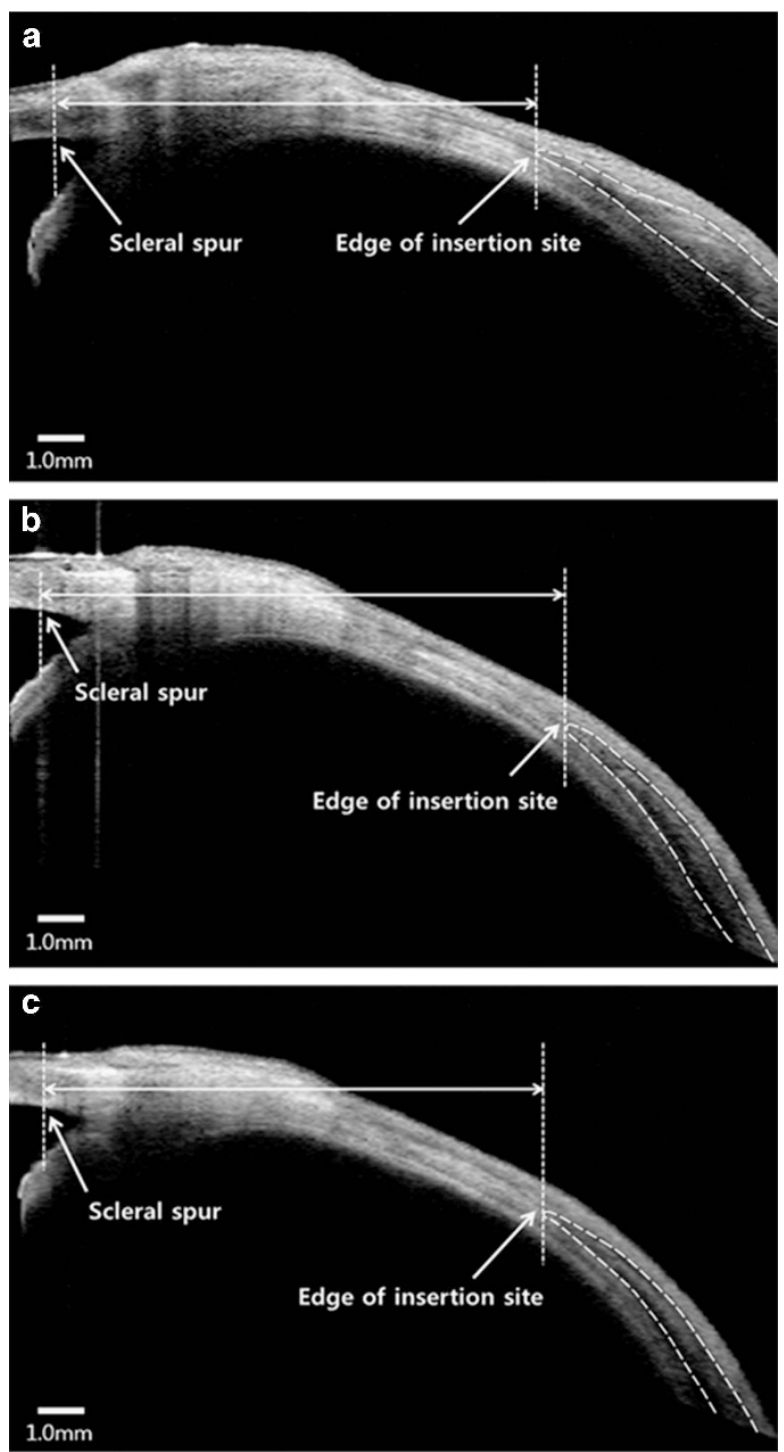

Figure 2 (a) Longitudinal changes in spur-insertion distance in lateral rectus muscle. AS-OCT image of postoperative 1 month, (b) postoperative 3 months, and (c) postoperative 6 months. With increasing time, we observed gradual resolution of swelling of the muscle and tendon tissue and were able to locate the new insertion site of the lateral rectus muscle.

\section{Results}

We enrolled 31 patients including 20 males and 11 females, were finally analyzed in this study. The mean age was $13 \pm 7$ years (range, 9-37 years), and mean follow-up was $208 \pm 8$ days. The mean angle of deviation was $26.0 \pm 5.9 \mathrm{PD}$ (range $15-40 \mathrm{PD}$ ). The mean amount of correction was $6.6 \pm 0.8 \mathrm{~mm}$ (range 6.0-8.0 mm). Four patients (13\%) underwent unilateral LR recession, and 27 patients $(87 \%)$ were underwent bilateral LR recession. All patients enrolled in this study had a satisfactory outcome, defining surgical success as distance ocular alignment at the primary position between 5 PD esodeviation and 10 PD exodeviation at 6 months after the operation.

\section{Longitudinal changes in each measurement}

The mean distance of spur-LR insertion measured preoperatively with AS-OCT was $5.5 \pm 0.7 \mathrm{~mm}$, compared with $11.1 \pm 0.9 \mathrm{~mm}$ at 1 month, $11.5 \pm 0.8 \mathrm{~mm}$ at 3 months, and $11.0 \pm 0.7 \mathrm{~mm}$ at 6 months postoperatively. The spurLR insertion measurements at 1,3 , and 6 months postoperatively were significantly increased compared with preoperative measurements (paired- $t$ test, all $P<0.001$; Figure 3). There were significant differences in spur-LR insertion between postoperative 1 and 3 months, and between 3 and 6 months (all $P<0.001$ ). However, the results did not show any significant difference in spur-LR insertion between postoperative 1 and 6 months $(P=0.413)$.

The mean preoperative deviation in the exotropia patients was $26.0 \pm 5.9 \mathrm{PD}$, compared to $0.9 \pm 1.8 \mathrm{PD}$ at 1 month, $0.7 \pm 1.4 \mathrm{PD}$ at 3 months, and $1.0 \pm 1.9 \mathrm{PD}$ at 6 months postoperatively. The angle of deviation at every postoperative visit was significantly decreased compared with the preoperative angle of deviation (paired- $t$ test, all $P<0.001)$. However, there was no significant difference in the angle of deviation between each postoperative visit $(P=0.165,0.810$, and 0.362 , respectively).The differences between preoperative and postoperative measurements of the spur-LR insertion distance were $5.6 \pm 1.0 \mathrm{~mm}$ at 1 month, $6.0 \pm 0.9 \mathrm{~mm}$ at 3 months, and $5.6 \pm 0.8 \mathrm{~mm}$ at 6 months. The differences between preoperative and postoperative angles of deviation were 25.0 $\pm 6.6 \mathrm{PD}$ at 1 month, $25.2 \pm 6.4 \mathrm{PD}$ at 3 months, and $25.0 \pm 6.6 \mathrm{~mm}$ at 6 months.

\section{Correlation between spur-LR insertion and surgical outcomes}

The overall spur-LR insertion measurement has a significantly negative correlation with postoperative amount of deviation (Pearson's correlation, $P=0.035$, $r=-0.218$ ). There was a significantly negative correlation between spur-LR insertion and postoperative amount of deviation at 6 months $(P=0.004, r=-0.467)$, but not at 1 and 3 months postoperatively. $(P=0.886$ and 0.432 respectively) (Figure 4).

The overall postoperative change in length of the spurLR insertion (the difference between preoperative and postoperative measurements), as measured by AS-OCT, was also significantly related to the change in amount of postoperative deviation (the difference between preoperative and postoperative measurements; $P=0.001$, $r=0.403$ ). There was a significantly positive correlation between those two measurements at 3 and 6 months 
a

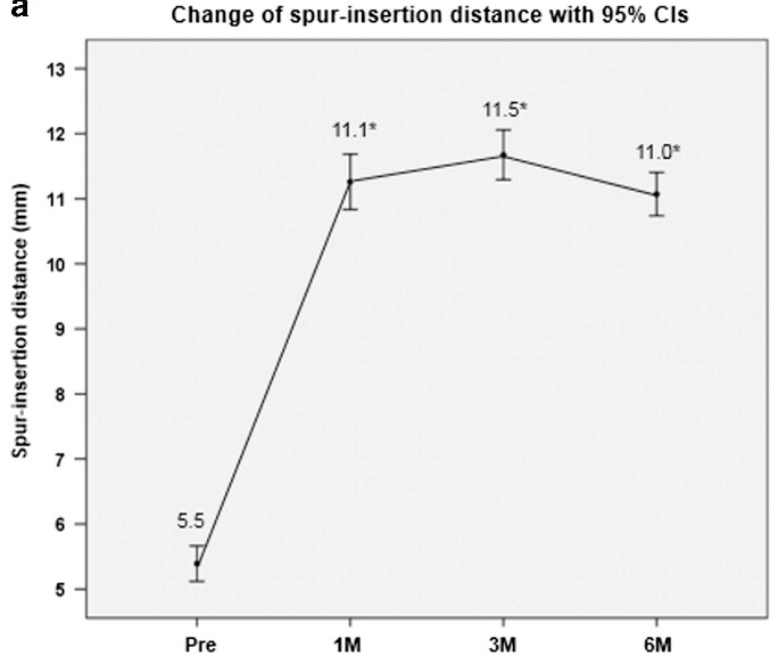

b

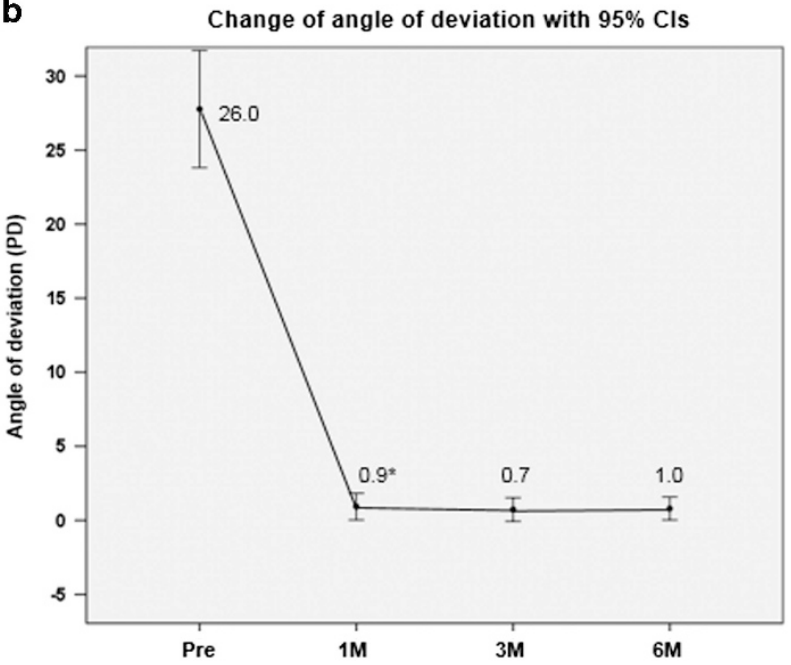

Figure 3 Postoperative changes in parameters. (a) Longitudinal changes in spur-insertion distance measured by AS-OCT after surgery. (b) Longitudinal changes in angle of deviation after surgery.
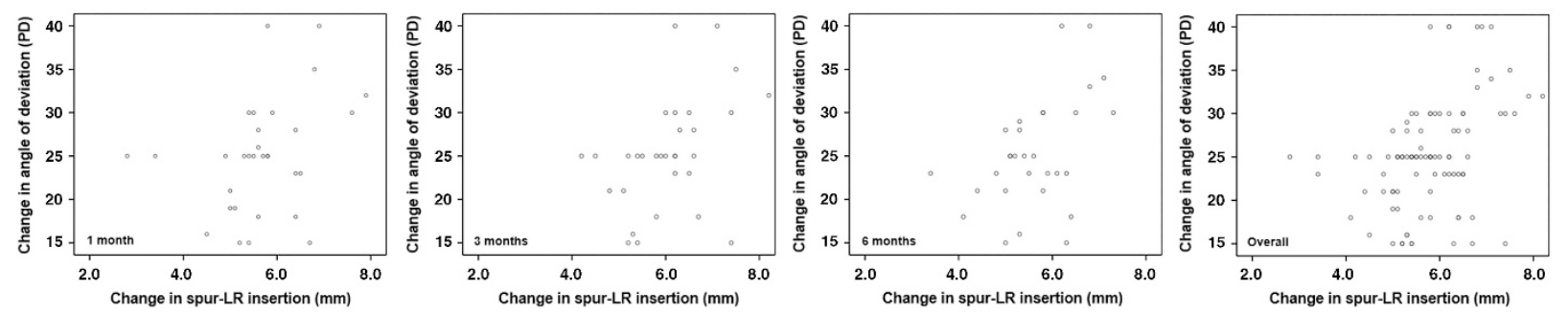

Figure 4 Correlation between postoperative spur-lateral rectus muscle (LR) insertion and angle of deviation. 'Change in spur-LR insertion' indicates difference value between postoperative and preoperative spur-LR insertion measurements. 'Change in angle of deviation' indicates difference value between postoperative and preoperative amount of deviation measurements.

$(P=0.020, r=0.415$, and $P=0.005, r=0.492$, respectively $)$, but not at 1 month postoperatively. $(P=0.068)$.

The intra-observer ICC value for reproducibility of AS-OCT measurements was 0.94 (95\% CI, 0.87-0.97), and the inter-observer ICC value was 0.83 (95\% CI, 0.67-0.92). The scatter plot showing the level of agreement between two observers is presented in Supplementary file 1. Change of the spur-LR insertion in each patient was presented in Supplementary file 2.

\section{Discussion}

Because strabismus surgery is primarily performed to change the insertion distance, knowledge of anatomic variations in extraocular muscle (EOM) location and correct measurement of the insertion distance are important aspects of preoperative planning. Furthermore, knowing the location of the new attachment site of EOM after strabismus surgery is important in determining the effect of strabismus surgery and is also a prerequisite for successful re-operation.
Therefore, there have been many trials showing the exact attachment site of EOM. Although EOM has been scanned with both static and dynamic magnetic resonance imaging, ${ }^{8-10}$ the distances from the limbus are difficult to measure from the resulting images. Patrick et al ${ }^{11}$ evaluated the EOM attachment site using an ultrasound biomicroscope during surgery and reported good agreement between the measurements noted during surgery and those measured with the ultrasound biomicroscope. However, measurement of the limbusinsertion distance is hindered by the fact that the horizontal limit of the caliper function of the ultrasound biomicroscope is $<5 \mathrm{~mm}$. ${ }^{11}$ In addition, ultrasound biomicroscope is a contact imaging technique and tear gel has to be used on the eye surface.

AS-OCT, which appears to be superior to other devices for studying EOM, was designed based on the principle of low-coherence interferometry using a superluminescent diode with a 1310-nm wavelength. It has the advantages of being a noncontact imaging modality for the anterior compartments of the eye at a specific meridian in the sitting position. Following a study by Liu et $a l^{5}$ that 
showed horizontal rectus muscle measurement with AS-OCT, our preliminary study evaluated the insertion site of horizontal rectus muscles using AS-OCT with varying eye positions in healthy normal adults. They used corneoscleral limbus which is also difficult to be obtained with AS-OCT as one of landmark in measuring insertion distance. We previously demonstrated that changes in eye position did not significantly affect the insertion distance measured by AS-OCT, but patient compliance was important in getting clear images. ${ }^{7}$ On the basis of these findings, therefore, we further investigated the preoperative and postoperative images of the lateral rectus muscle, which can be imaged more clearly using AS-OCT

The posterior face of the insertion site was clear preoperatively but became unclear postoperatively because of tissue edema. Preoperatively, we used the posterior face of the insertion, which is easily identified in the images. On the other hand, for accuracy of postoperative measurement, we used the anterior face of the insertion site to evaluate longitudinal changes in the insertion site. This could be found at the anterior edge of tissue edema in postoperative images and was therefore relatively unaffected by tissue edema. In addition, we also used the spur-LR insertion distance for accuracy. Because the scleral spur was not affected by tissue edema and gave a clearer image, it became a better landmark for continuous measurements of LR insertion distance than the corneoscleral limbus, which was not observed clearly in postoperative images.

In this study, most of the postoperative insertion distances measured by AS-OCT were shorter than the predicted location of LR insertion after surgery. This can be explained by a difference in the landmark for each measurement. The predicted location was the shortest distance between the limbus and posterior face of insertion site, whereas AS-OCT measurement was the shortest distance between the spur and anterior face of insertion site. These different landmarks could lead to the discrepancy between these two measurements in this study.

Consequently, there was no significant movement between postoperative 1 and 6 months. This finding might reflect tissue swelling of raw muscle material attached to the sclera, which could affect the images obtained with AS-OCT. It was difficult to identify the attachment site of the rectus muscle 1 week postoperatively because tissue swelling around the attachment site obscured visualization of the new insertion. It was also difficult to distinguish the anterior edge and posterior edge of the rectus muscle after surgery because of remaining tissue swelling. Therefore, we investigated the anterior edge of rectus muscle insertion, which was more easily visualized in AS-OCT images during the postoperative follow-up period. Tissue swelling decreases slowly after surgery, and the tissue volume around the new insertion site also decreases over time. The new insertion sites of LR muscles were observed clearly after 3 months postoperatively. This might explain the early movement of tissue observed in AS-OCT images and also suggests that the movement was not a real displacement of the rectus muscle. Further study to verify it is required.

We found that a significant relationship between LR insertion distance measured in AS-OCT and postoperative amount of deviation. Regarding with each postoperative visit, these two measurements showed a significant correlation at 6 months postoperatively. This finding might reflect that AS-OCT might be a useful tool not only for finding the insertion site of horizontal muscle, but also for tracking the sites after surgery and determining the effect of strabismus surgery. However, caution should be used in tracking insertion site in early postoperative period based on the results of this study.

This study has some limitations. First, our sample size was small and the follow-up period was relatively short. Sample size in this study was small for method agreement study, and 6 months of follow-up might be insufficient to observe all changes in the insertion site. A study with larger sample size and longer-term follow-up is needed. In addition, we used patients from a single center for this study and all patients had the same ethnicity. Hence, some of the results may not be valid for other ethnic groups. Second, we included only conventional method of recession surgery. We could not compare the movement of rectus muscles between the two different methods including conventional and hang-back method. Further well controlled study is required. Third, postoperative insertion site with larger recession over $8 \mathrm{~mm}$ was not easily detected. It depended on patient cooperation, but cooperation was poor particularly in children. Therefore, we recommended a reliable use of AS-OCT in evaluating postoperative insertion in cases of recession $\leq 8 \mathrm{~mm}$. At last, there were inter-observer differences, although excellent inter-observer reliability was shown in this study. The main contributor to interobserver differences is variability in identifying the location of the attachment site of the LR muscle. Therefore, the anterior and posterior faces of the insertion site were used in respective circumstances to minimize the variation derived from the edema effect. Finally, we failed to evaluate changes in EOM insertion distance other than LR muscle because we could not obtain clear images of other EOMs with AS-OCT. Further controlled studies are required to assess the insertion site according to surgical type or other rectus muscles with a larger sample size. 
Despite these limitations, this is the first study to evaluate the longitudinal change in rectus muscle movement with AS-OCT. We suggest that AS-OCT could be a useful instrument to evaluate the insertion of EOM preoperatively, and also has potential application for understanding postoperative changes in EOM after tissue swelling subsides.

\section{Summary}

What was known before

- AS-OCT can be used to image the structure of the horizontal rectus muscles and shows good agreement with intraoperative measurements.

What this study adds

- We provided more accurate method to evaluate muscle insertion when using AS-OCT, and AS-OCT has potential application for understanding postoperative changes in extraocular muscle after tissue swelling subsides.

\section{Conflict of interest}

The authors declare no conflict of interest.

\section{Author contributions}

JL and SO designed and conducted the study. JL and IL collected the data. JL analyzed and interpreted the data, drafted and revised the manuscript. KP contributed to the review and revision of the manuscript. SO approved the final version of the manuscript.

\section{Acknowledgements}

Statistician: Kyung Ah Kim, Ph.D., Department of Biomedical Statistics, Samsung Medical Center, Sungkyunkwan University School of Medicine, Seoul, Republic of Korea. A grant of the Korea Healthcare technology R\&D Project, Ministry of Health and Welfare, Republic of Korea. (HI10C2020).

\section{References}

1 Mazow ML, Leal V. Experimental creeping muscle. J Pediatr Ophthalmol 1976; 13: 168-170.

2 Ohtsuki H, Oshima K, Hasebe S, Kobashi R, Okano M, Furuse T. Extraocular muscle surgery in a rabbit model: site of reattachment following hang-back and conventional recession. Graefes Arch Clin Exp Ophthalmol 1994; 232: 689-694.

3 Fink WH. A study of the anatomical variations in the attachment of the oblique muscles of the eyeball. Trans Am Acad Ophthalmol Otolaryngol 1947; 51: 500-513.

4 Repka MX, Fishman PJ, Guyton DL. The site of reattachment of the extraocular muscle following hang-back recession. J Pediatr Ophthalmol Strabismus 1990; 27: 286-290.

5 Liu X, Wang F, Xiao Y, Ye X, Hou L. Measurement of the limbus-insertion distance in adult strabismus patients with anterior segment optical coherence tomography. Invest Ophthalmol Vis Sci 2011; 52: 8370-8373.

6 Ngo CS, Smith D, Kraft SP. The accuracy of anterior segment optical coherence tomography (AS-OCT) in localizing extraocular rectus muscles insertions. J AAPOS 2015; 19: 233-236.

7 Park KA, Lee JY, Oh SY. Reproducibility of horizontal extraocular muscle insertion distance in anterior segment optical coherence tomography and the effect of head position. J AAPOS 2014; 18: 15-20.

8 Ozgen A, Aydingoz U. Normative measurements of orbital structures using MRI. J Comput Assist Tomogr 2000; 24: 493-496.

9 Tian S, Nishida Y, Isberg B, Lennerstrand G. MRI measurements of normal extraocular muscles and other orbital structures. Graefes Arch Clin Exp Ophthalmol 2000; 238: 393-404.

10 Demer JL, Kerman BM. Comparison of standardized echography with magnetic resonance imaging to measure extraocular muscle size. Am J Ophthalmol 1994; 118: 351-361.

11 Watts P, Smith D, Mackeen L, Kraft S, Buncic JR, Abdolell M. Evaluation of the ultrasound biomicroscope in strabismus surgery. J AAPOS 2002; 6: 187-190.

Supplementary Information accompanies this paper on Eye website (http://www.nature.com/eye) 